CERN/SI-Int/BR-72-4

1 September 1972

\title{
SENSITIVITY OF A CLASS OF DYNAMIC SYSTEMS
}

WITH RESPECT TO A SMALL DELAY

I. Gumowski

CERN, Geneva, Switzerland

Paper submitted to the

3rd IFAC Symposium on Sensitivity, Adaptivity, and Optimality

Ischia, 18-21 June 1973

GENEVA

1972 



\section{SENSITIVITY OF A CLASS OF DYNAMIC SYSTEMS}

WITH RESPECT TO A SMALL DELAY

\section{Gumowski}

CERN, Geneva, Switzerland

\section{INTRODUCTION AND STATEMENT OF THE PROBLEM}

In feedback loops of dimensionally large systems the signal propagation time c around the loop may become non-negligible compared to the inverse of the loop bandwidth. Such a situation exists, for example, in several particle accelerators, which use feedback to control some dynamic properties of the particle beam in the accelerator ring. The length of connecting cables may run into hundreds of metres, which leads to a delay of several microseconds, the loop bandwidth being of the order of $50 \mathrm{kHz}$.

The analysis of a dimensionally large feedback loop is usually carried out in two stages. In the first stage it is supposed that the signal propagation time is qualitatively unimportant. The feedback loop is therefore described by a usual linear or non-linear dynamic system

$$
\dot{x}(t)=f(x, t), t \geqslant t_{0}, x\left(t_{0}\right)=x_{0}
$$

Unless otherwise specified, all functions and constants are real-valued in (1), as well as in all subsequent equations. Sufficient smoothness is assumed for the validity of the implied existence and uniqueness theorems, except when the argument pertains specifically to the problem of sensitivity, where the amount of continuity is specified explicit1y. In the second stage the signal propagation time is taken into account, and instead of (1) one obtains

$$
\begin{aligned}
& \dot{x}(t)=g(x(t), x(t-\alpha), t), t \geqslant t_{0} \\
& x(t)=\theta(t), t_{0}-\alpha \leq t \leq t_{0},
\end{aligned}
$$

where $\theta(t)$ represents the signal "stored" in the cables and other memory elements. Since by definition (1) and (2) describe the same physical system, these equations should be continuous with respect to the parameter $c$ and "close" when $c$ is small. The continuity requires in particular

$$
\lim _{c \rightarrow 0} g(x(t), x(t-c), t)=f(x, t) \text {. }
$$

Knowing that (1) and (2) are close leads to the basic problem of determining under what conditions the corresponding solutions are qualitatively similar and quantitatively close.
A RELATION BETWEEN SENSITIVITY AND THE THEORY OF ASYMPTOTIC EXPANSIONS

A partial answer to the problem of closeness of the solution of (1) and (2) is supplied by the usual sensitivity theory, when $c$ is interpreted as a parameter variation from the reference value $c=0$. The study of the sensitivity coefficient $\partial x / \partial c$ may be considered as a particular case of the study of converging or asymptotically converging expansions $\left.\left.{ }^{1}\right)^{*}\right)$. For the latter there exists extensive literature (for example, Refs. 2-5). In order to illustrate the mutual relationship, consider a typical theorem of the asymptotic method (Ref. 4, p. 135).

If Eq. (2) is reducible to the standard small parameter form

$$
\begin{aligned}
& \dot{x}(t)=\varepsilon X(x(t), x(t-c), t), t \geqslant 0, \\
& x(t)=\theta(t),-c \leqslant t \leqslant 0, \quad \theta(0)=x_{0},
\end{aligned}
$$

where $0<\varepsilon<1$, X is a sufficiently smooth function with respect to $c, x(t), x(t-c)$, and there exists the limit function

$$
X_{0}(x)=\lim _{T \rightarrow \infty} \int_{0}^{T} X(x, x, t) d t,
$$

then the solution of the "generating system"

$$
\dot{y}(t)=\varepsilon X(y(t), y(t), t), y(0)=x_{0}
$$

and of the "averaged system"

$$
\dot{\xi}(t)=\varepsilon X_{0}(\xi), \quad \xi(0)=x_{0},
$$

are both close to the solution of the initial system (4). In particular, for any pair of values $n>0$, $t_{1}>0$ there exists an $\varepsilon_{0}$ such that

$$
\begin{gathered}
\|x(t)-y(t)\|<\varepsilon a L\left(A+M t_{1}\right) e^{2 L t_{1}}<\eta \\
0 \leqslant t \leqslant \varepsilon^{-1} t_{1},
\end{gathered}
$$

\footnotetext{
*) "Superior numbers refer to similarly-numbered references at the end of this paper."
} 
provided $0<E<E_{0} ; \quad L, A$, and $M$ are known positive constants. More specifically, L is a Lipschitz constant of $x(x, y, t), M$ is the bound of its norm, and $A$ is a measure of the compatibility of the shape of the memory function $\theta(t)$ with the shape of $x(t)$. The smallness of the norm in (8), i.e. the closeness of $x(t)$ and $y(t)$, is assured by the smallness of the product $\varepsilon$ c L.

Let us introduce an auxiliary real parameter $\delta$ such that $|\delta|=\varepsilon c$ L. From the examination of particular cases it will be seen that $\delta$ represents an "effective" delay of (2), and that $|\delta|$ need not be small simultaneously with the real delay $c$. A situation when a smal1 c does not imply a sma $11|\delta|$ occurs precisely in the case of dimensionally large feedback loops with appreciable bandwidth. The inequality (8) thus becomes inapplicable to the solutions of (1) and (2), and new problems arise. The simplest of these problems is the determination of bifurcation values of $\delta$. The first bifurcation occurs when $x(t)$ and $y(t)$ become qualitatively different, rendering the estimate (8) inappropriate in principle. In this paper the possible bifurcations will be studied in a restricted class of systems (1), (2).

\section{DEFINITION OF THE CLASS}

The equations considered are either particular cases or slight modifications of the general phase-lock equation ${ }^{6}$ ):

$$
n \phi+k H(s) \cdot F(\phi)=\omega
$$

where $\phi$ is the state variable (phase), s the operator $\mathrm{d} / \mathrm{dt}, \mathrm{H}(\mathrm{s})$ is a rational function in s verifying $\mathrm{H}(0)=1$ (normalized filter transfer function), $\mathrm{F}(\phi)$ is a periodic function of period $2 \pi$ (normalized discriminator characteristic), $k$ is a positive constant (nominal bandwidth of the phase loop) and $\omega$ is a constant or a function of time (instantaneous frequency error). When the phase loop possesses a non-negligible signal propagation time $c$, the formal equivalent of (9) is

$$
\partial \phi+k H(s) \cdot F\left(e^{-c s} \phi\right)=\omega \text {. }
$$

The property $F(\phi)=F(\phi+2 \pi)$ allows the representation of the solutions of (9) in a cylindrical phase space. Since the solutions of (10) depend on an initial function $\theta(t)$ and not just on an initial point, they must be studied in a rather general functional phase space. In this paper only a subspace of this functional space is considered, consisting of functions which are sufficiently smooth with respect to $t$ and the parameter $c$. It would be convenient to require analyticity in $t$ and $c$, but this requirement turns out to be too stringent in many cases of practical interest. It is obvious that subject to analyticity an otherwise arbitrary initial function

$$
\phi(t)=\theta(t), t_{0}-c \leqslant t \leqslant t_{0}
$$

is equivalent to an initial value $\phi\left(t_{0}\right)=\theta\left(t_{0}\right)$, and the solutions of (10) become representable in an ordinary cylindrical phase space. In the absence of analyticity, sufficient smoothness will be assumed to preserve at least an approximate representability in a cylindrical phase space. It will be shown that such a smoothness condition does not unduly restrict the scope of possible bifurcations of (10).

Following the usual practice, the properties of (9), (10) will be studied by looking first for singular solutions, describing constant, periodic, and almostperiodic steady states. The existence conditions and the stability of solutions will be of particular interest. Whereas the former can often be expressed analytical1y, the latter invariably require a numerical approach. Let $\mathrm{m}, \mathrm{n}$ be the degrees of the polynomials in the numerator and denominator of $\mathrm{H}(\mathrm{s})$, respectively. When $m \leq n$, the equations (9), (10) are easily transformed to the form (1), (2). The resultant (differential) order is $n+1$. The study of the bifurcations of (10) requires, of course, that the solutions of (9) are already known. Unfortunately this is only the case for $\mathrm{n}=0$ and to some extent for $\mathrm{n}=1$, when $\mathrm{F}(\phi)$ is of a relatively simple form.

\section{BIFURCATIONS OF A LINEAR EQUATION WITH DELAY}

The simplest possible form of (10) is obtained with the assumptions $H(s) \equiv 1,|\phi| \ll 2 \pi$, allowing one to write $\mathrm{F}(\phi)=\phi$ and

$$
\dot{\phi}(t)+k \phi(t-k)=\omega(t) \text {. }
$$

Because of linearity the basic properties of (12) are not affected by $\omega(t)$; it will thus be assumed that u(t) has been eliminated with the help of a particular solution. If in addition $t$ is replaced by kt, (12) reduces to the one-parameter form

$$
\dot{\phi}(t)=-\phi(t-\delta) \quad, \quad \delta=k c
$$

It should be noted that $c>0$ implies $\delta>0$ for the class of equations chosen. In general the real delay $c$ and the effective delay $\delta$ need not be of the same sign. Since $\varepsilon=1, L=1$ in (13), the standard form (4) can only be obtained when $\delta$ is sufficiently small. The linear equation (13) is thus already appropriate For the study of some bifurcations due to a variation of $\delta$. No loss of generality occurs by letting $t_{0}=0$. The generating equation for the purpose of sensitivity is

$$
\dot{\phi}(t)=-\phi(t)
$$

It is well known that the unique solution of (13), defined by the initial condition (11), with an integrable $\theta(t)$, is given by

$$
\begin{aligned}
& \phi(t)=\theta(t), \quad-\delta \leq t \leq 0 \\
& \phi(t)=\theta(0)-\int_{0}^{t} \phi(r-\delta) d r, \quad 0 \leq t \leq \delta, \\
& \phi(t)=\theta(\delta)-\int_{0}^{t} \phi(r-\delta) d r, \delta \leq t \leq 2 \delta, \\
& \cdots \cdots \cdots
\end{aligned}
$$

When $\theta(t)$ is not already analytical, the smoothness of $\phi(t)$ increases with $t$, i.e. the order of continuous $\left(s^{n} \phi\right)$ increases as $n \approx t / \delta$. The qualitative properties of (15) depend, of course, also on the value of $\delta$. It is known that (13) admits a unique family of solutions which are analytical both in $t$ and in $\delta$ in the neighbourhood of $\delta=0$ ):

$$
\phi(t)=\phi(0)\left(\sum_{n=0}^{\infty} \frac{n^{n}}{n !} \delta^{n}\right)^{-1} \sum_{n=0}^{\infty} \frac{(n \delta-t)^{n}}{n !} \text {. }
$$


The one-parameter family $(16)$ is thus a natural extension for $\delta \neq 0$ of the analytical generating solution

$$
\phi(t)=\phi(0) \sum_{n=0}^{\infty} \frac{(-t)^{n}}{n !}=\phi(0) \cdot e^{-t}
$$

of (14). Unfortunately, the series in (16) converge only when $|\delta|<1 / e$. It is readily possible to extend the function defined by (16) to $\delta \leq-1 / e$ (p. 79-84, vol. 4 of $\operatorname{Ref} 8$ ), but the method used fails for $\delta \geq 1 /$ e.

An extension beyond $\delta=1 /$ e can, however, be made on the basis of two additional properties of (13)-(16). The first property is the identity

$$
\left(\sum_{n=0}^{\infty} \frac{n^{n}}{n !} \delta^{n}\right)^{-1} \sum_{n=0}^{\infty} \frac{(n \delta-t)^{n}}{n !}=e^{\gamma t},
$$

where $Y$ is the unique real root of the characteristic equation

$$
\gamma+e^{-\delta \gamma}=0
$$

of (13) when $|\delta|<1 / e^{7)}$. The second property is derived from the nature and distribution of real and complex roots $\gamma_{k}$ of (19) for any real $\delta$, and the representability of an arbitrary function $\theta(t)$ in (11) by means of the corresponding eigenfunctions of (13). It is known ${ }^{9-11}$ ) that the roots of (19) are enumerable and that they can be ordered according to their moduli, i.e. so that $\left|\gamma_{k+1}\right|>\left|\gamma_{k}\right|$. The exponent $\gamma$ in (18) can be defined alternatively by means of

$$
|r|=\min _{k}\left|r_{k}\right| .
$$

An arbitrary integrable function $\theta(t)$ can be represented in the interval $-\delta<t<0$ by

$$
\theta(t)=\sum_{k=-\infty}^{+\infty} C_{k} e^{\gamma_{k} t},
$$

where

$$
C_{k}=\frac{\gamma_{k}}{1+\delta \gamma_{k}}\left[\int_{-\tau}^{0} e^{-\gamma_{k} \tau}, \theta(\tau) d \tau+\frac{1}{\gamma_{k}} \theta(0)\right] .
$$

The expansion coefficients $C_{k}$ results from the orthogonality relation

$$
\int_{-\tau}^{0} e^{-\gamma_{k} t} \cdot e^{\gamma_{m} t} d t+\frac{1}{\gamma_{k}}=\left\{\begin{array}{cl}
\left(1+\delta \gamma_{k}\right) / r_{k} & , k=m \\
0 & , k \neq m
\end{array}\right\} .
$$

From (21) and (15) it is possible to obtain expressions of the form (21) for any interval

$(\mathrm{n}-1) \delta<\mathrm{t}<\mathrm{n} \delta, \mathrm{n}=0,1, \ldots$. The analytical solution (16), valid for $|\delta|<1 / \mathrm{e}$ and any $t$, corresponds to the set of initial functions $\theta(t)$ defined by $\mathrm{C}_{\mathrm{k}}=\phi(0)$ if $\mathrm{k}=1, \mathrm{C}_{\mathrm{k}}=0$ if $\mathrm{k} \neq 0$, and is given by

$$
\phi(t)=\phi(0) \cdot e^{\gamma t}
$$

The existence of an extension of (16) to $\delta<-1 / e$ implies the validity of (24) for $-\infty<\delta<1 /$ e. This conclusion is comfirmed by the existence of the required real root of (19). It is therefore natural to use (24) also as an extension of (16) for $\delta 21 /$ e. A fundamental difficulty is encountered at this point, because the simple real root $\gamma$ of (19) no longer exists when $\delta \geq 1 / \mathrm{e}$. In fact, $\gamma$ turns into a double real root when $\delta=1 / \mathrm{e}$, which in turn splits into two simple complex conjugate roots when $\delta>1 / e$. This fundamental difficulty can only be removed by assuming that the extension of the analytical solution (16), valid for any $\delta \neq 1 / \mathrm{e}$, is of the form

$$
\begin{aligned}
& \phi(t)=e^{\alpha t}(A \cos \beta t+B \sin \beta t) \\
& \gamma=\alpha=i \beta
\end{aligned}
$$

where A, B are "integration constants", and $\gamma$ is given by (19), (20). In other words, in the space of (extended) analytical solutions the differential equation of order one with delay (13) behaves like a differential equation without delay of order one when $\delta<1 / e$, and like a differential equation without delay of order two when $\delta \geq 1 /$ e. The first bifurcation introduced by a smal1 delay $c$ thus consists in an increase of the "effective" differential order.

For (13) the first bifurcation value in $\delta=\delta_{0}=1 / \mathrm{e}$, because $\beta=0$ in (25) when $\delta<1 / e$ and $\beta>0$ when $\delta>1 /$ e. The values of $\gamma$ are easily computed (Table 1). A second bifurcation of (13) occurs when $\delta=\delta_{1}=\pi / 2$, because $\alpha<0$ if $\delta<\pi / 2, \alpha=0$ if $\delta=\pi / 2$, and $\alpha>0$ if $\delta>\pi / 2$. The corresponding bifurcation solution is periodic and purely sinusoidal of period $2 \pi$. Because of the second bifurcation the first order equation with delay

$$
\dot{x}(t)+k x(t-k)=0, c=\frac{\pi}{2 k},
$$

whose analytic solution is

$$
x(t)=A \cos k t+B \sin k t
$$

is also a generating equation for a class of quasilinear oscillatory phenomena, in the same right as the ordinary equation

$$
\ddot{x}(t)+a \dot{x}(t)+k^{2} x(t)=0, a=0 .
$$

Comparing the damping rates and zero-crossing abscissae of an oscillatory solution it is generally possible to tell whether it derives from (26) or (28) 12,13 ).

Bifurcations involving an increase of the effective differential order occur also in linear differential equations with delay of a higher order, because as a rule the presence of the factor $e^{-c s}$ in the characteristic equation leads to the splitting of a simple real root into two complex conjugate ones for at least one $c=c_{0}$. The validity of the asymptotic estimate ( 8 ) is obviously limited to values of $c$ smaller than the bifurcation value $c_{0}$.

\section{CONSTANT STEADY STATES}

The constant steady states of (2) are described, as usual, by, the real roots $x$ of

$$
g(x, x, t)=f(x, t)=0 .
$$


In most cases (29) has no roots (independent of $t$ ), unless $\partial f / \partial t \equiv 0$. The stability of a known steady state can be determined locally by means of the variational equation and globally by the second method of Liapunov. The variational equations are linear differential equations with delay, and in the second method V-functions are replaced by Vfunctiona1s.

As an illustration consider (10) when $\mathrm{H}(\mathrm{s}) \equiv 1$, $F(\phi)=\sin \phi$, and $\omega$ is a constant $\omega_{0}$. With these assumptions (10) becomes

$$
\dot{\phi}(t)+k \sin \phi(t-c)=\omega_{0}
$$

On the phase cylinder $\phi, \psi=\dot{\phi}$ there exist two roots

$$
\begin{aligned}
& \phi_{1}=\arcsin a_{0}, \phi_{2}=\pi-\phi_{1}, \\
& \psi_{1}=\psi_{2}=0, a_{0}=\omega_{0} / k,\left|a_{0}\right|<1 .
\end{aligned}
$$

Replacing $\mathrm{t}$ by $\mathrm{kt}$ and linearizing around $\left(\phi_{1}, \psi_{1}\right)$ yields the variational equation

$$
\dot{\phi}(t)+k \sqrt{1-a_{0}^{2}} \cdot \phi(t-x)=0 \text {. }
$$

The steady state $\phi(t)=\phi_{1}$ is thus asymptotically stable when

$$
\delta=k \propto \sqrt{1-a_{0}^{2}}<\frac{\pi}{2}
$$

The steady state $\phi(t)=\phi_{2}$ turns out to be unstable. By means of one particular $\mathrm{V}$-functional it has been found ${ }^{14}$ ) that $\phi(t)=\phi_{1}$ is asymptotically stable for arbitrary initial $\theta(t)$ in a certain metric space provided

$$
k_{x} \leqslant 0.82\left(1-\left|a_{0}\right|\right) \text {. }
$$

Numerical tests have shown ${ }^{15}$ ) that (34) is quite pessimistic in practice, i.e. it overemphasizes certain shapes of $\theta(t)$ which are not typical in a physical context. In order to improve (34) systematically, it is necessary to have an explicit definition of the domain of influence of a steady state in terms of the properties of the admissible $\theta(t)$. No such definition is at present available. It is possible, however, to estimate the size of the domain of influence on the phase cylinder if some nonconstnat, autonomous, asymptotically stable steady states are known.

\section{AUTONOMOUS PERIODIC STEADY STATES}

It has been known for some time that non-linear, autonomous differential equations of order one with delay possess periodic solutions. The earliest examples appear to be $\left.{ }^{16-1 \theta}\right)$

$$
\begin{aligned}
& \dot{x}(t)=-k[1+x(t)] x(t-c), k_{c}>\frac{\pi}{2}, \\
& \dot{x}(t)=-k\left[1+x^{2}(t)\right] x(t-c), k c>\frac{\pi}{2}, \\
& \dot{x}(t)=-x(t-g), g=c+k_{2} x^{2}(t), k>\frac{\pi}{2} .
\end{aligned}
$$

It was found numerically ${ }^{18}$ ) that the shapes, amplitudes and periods of the periodic solutions of (37) do not depend on the shape of the initial function in (11). For a fixed $c>\pi / 2$ and $c_{2}<0$ the perio- dic solutions of (37) are unique and asymptotically stable. These periodic solutions are therefore analoguous to the usual periodic solutions described by limit cycles. For the sake of brevity they will be called of limit-cycle type. It turns out that the periodic solutions of (35) and (36) are also of limit-cycle type $17-20)$. In fact, at present it is safe to conjecture that periodic solutions of limitcycle type are more a rule than an exception in nonlinear autonomous equations with delay.

Numerical evidence shows that autonomous periodic solutions are frequently almost sinusoïdal when the effective delay is close to $\pi / 2$ (Figs, 1-10). It should therefore be possible to find these solutions by means of the small parameter method of Poincaré. The small parameter approach turns out to be successful, provided the order of magnitude of the nonlinearity and the form of the solution are appropriately chosen. Owing to the absence of a general existence theorem, there are still no systematic rules for making this choice. Comparison with computer results constitutes a useful consistency check. Once the correct orders of magnitude have been found, they appear to be physically self-evident and are easily justified a posteriori.

As an illustration the periodic solutions of (30) will be determined. Consider first the somewhat simpler equation

$$
\begin{aligned}
& \dot{x}(t)=-\left(\frac{\pi}{2}+a\right) \cdot P\left(x_{t}\right) \cdot x(t-1) \\
& P\left(x_{t}\right)=1+b x(t-1)+c x^{2}(t-1)+d x^{3}(t-1)
\end{aligned}
$$

where $a, b, c$, and $d$ are known constants. If the small parameter is again designated by $\varepsilon$, it is necessary to assume

$$
\begin{array}{ll}
\tau=r_{0} t, & \tau_{0}=\frac{\pi}{2} \sum_{i=0}^{\infty} h_{i} \varepsilon^{i}, \\
a=\varepsilon^{2} \alpha, & x(t)=\sum_{i=0}^{\infty} \varepsilon^{i+1}, x_{i}(r)
\end{array}
$$

where $h_{0}=1$ and $h_{i}, i>0$, are undetermined constants. The definition of $\tau_{0}$ represents the usual transformation ${ }^{21}$ ) of the initially unknown period $T$ in $t$ to the period $2 \pi$ in $\tau$.

A short exploratory analysis with $\max i=1$ shows that $h_{1}=0$. This first step is unnecessary in principle; it simply makes it possible not to write out explicitly many vanishing terms. As usual, the substitution of (39) into (38) converts the latter into a form which can be solved recursively:

$$
\begin{aligned}
\varepsilon \dot{x}_{0}(\tau) & +\varepsilon^{2} \dot{x}_{1}(\tau)+\varepsilon^{3} \dot{x}_{2}(\tau)+\cdots= \\
= & -\left(1+\frac{2 \alpha}{\pi} \varepsilon^{2}\right)\left(1+h_{2} \varepsilon^{2}+\cdots\right) \cdot P(y) \cdot y(\tau), \\
P(y)= & 1+b y(\tau)+c y^{2}(\tau)+d y^{3}(\tau), \\
y(\tau)= & \varepsilon x_{0}\left(\tau-\frac{\pi}{2}\right)+\varepsilon^{2} x_{1}\left(\tau-\frac{\pi}{2}\right)+ \\
& +\varepsilon^{3}\left[x_{2}\left(\tau-\frac{\pi}{2}\right)+\frac{\pi}{2} h_{2} \dot{x}_{0}\left(\tau-\frac{\pi}{2}\right)\right]+\cdots . .
\end{aligned}
$$

Similarly to the case without delay one looks for an (extended) analytical periodic solution satisfying 
the condition $\dot{x}_{j}(0)=0$. Equating in (40) the coefficients of $E^{i}, i=1,2$ yields

$$
\begin{gathered}
\dot{x}_{0}(\tau)+x_{0}\left(\tau-\frac{\pi}{2}\right)=0, x_{0}(\tau)=A_{0} \cos \tau . \\
\dot{x}_{1}(\tau)+x_{1}\left(\tau-\frac{\pi}{2}\right)=-b x_{0}^{2}\left(\tau-\frac{\pi}{2}\right),
\end{gathered}
$$

where $A_{0}$ is an undetermined constant. It can be verified by substitution that the solution of (42) is

$$
\begin{aligned}
x_{1}(\tau) & =A_{4} \cos \tau-\frac{2}{5} b A_{0}^{2} \sin \tau+ \\
& +\frac{b}{10} A_{0}^{2}(-5+2 \sin 2 \tau-\cos 2 \tau)
\end{aligned}
$$

where $A_{1}$ is also an undetermined constant. From the coefficients of $E^{3}$ one finds

$$
\begin{aligned}
\dot{x}_{2}(\tau) & +x_{2}\left(\tau-\frac{\pi}{2}\right)=-\left[\left(h_{2}+\frac{2 \alpha}{\pi}\right) x_{0}\left(\tau-\frac{\pi}{2}\right)+\right. \\
& +2 b x_{0}\left(\tau-\frac{\pi}{2}\right) \cdot x_{1}\left(\tau-\frac{\pi}{2}\right)+ \\
& \left.+\frac{\pi}{2} h_{2} \dot{x}_{0}\left(\tau-\frac{\pi}{2}\right)+2 x_{0}^{3}\left(\tau-\frac{\pi}{2}\right)\right]= \\
& =\left[\left(h_{2}+\frac{2 \alpha}{\pi}\right) A_{0}+\left(\frac{3}{4} c-\frac{11 b^{2}}{10}\right) A_{0}^{3}\right] \cos \tau+ \\
& +\left[\frac{b^{2}}{5} A_{0}^{2}-\frac{\pi}{2} h_{2}\right] A_{0} \sin \tau+\cdots
\end{aligned}
$$

The necessary condition for the existence of a periodic solution of (38) is the absence of secular terms in (40). The coefficients of $\cos \tau$ and $\sin \tau$ in the right-hand side of (44) must therefore vanish. The two algebraic equations so obtained yield

$$
A_{0}=\left(\frac{40 \alpha}{2(11 \pi-4) b^{2}-15 \pi c}\right)^{\frac{1}{2}}, \quad h_{2}=\frac{2 b^{2} A_{0}^{2}}{5 \pi} .
$$

Continuing the recursive process to $\varepsilon^{4}$ it is possible to determine uniquely the constants $A_{1}, h_{3}$, and so on, until the desired number of terms in (39) is attained. It should be noted that neither the generating amplitude $A_{0}$ nor the frequency deviation $h_{2} \varepsilon^{2}$ depend on the coefficient $d$ of $x^{4}(t-1)$ in (38). The $\varepsilon$-dependence of (39) ensures the same result for any term $x^{i}(t-1), i \geq 4$. The solution (39), (45) therefore holds in principle also for equation (30). In fact, replacing $k$ by $k t$ and $\phi$ by $x-\phi_{1}$ transforms (30) into

$$
\begin{aligned}
& \dot{x}(t)=-k_{x} \sqrt{1-a_{0}^{2}} \cdot P\left(x_{t}\right) \cdot x(t-1) \\
& P\left(x_{t}\right)=1-\frac{a_{0}}{2 \sqrt{1-a_{0}^{2}}} x(t-1)-\frac{1}{6} x^{2}(t-1)+\cdots
\end{aligned}
$$

In order to use (39), (45) it is sufficient to let

$$
a=c-\pi\left(2 k \sqrt{1-a_{0}^{2}}\right)^{-1}
$$

In the original variables the periodic solution is

$$
x(t)=\arcsin \frac{\omega_{0}}{k}+A \cos \frac{t}{T_{0}}+\cdots,
$$

where

$$
\begin{aligned}
& A=\left(\frac{80 k\left(k^{2}-\omega_{0}^{2}\right)-40 \pi}{(6 \pi-4) \omega_{0}^{2}+5 \pi k^{2}}\right)^{\frac{1}{2}} \\
& \frac{1}{T_{0}}=\frac{5 \pi^{2}\left(k^{2}-\omega_{0}^{2}\right)}{10 \pi\left(k^{2}-\omega_{0}^{2}\right)+\omega_{0}^{2} A^{2}+\cdots},
\end{aligned}
$$

The periodic solution (48) is asymptotically stable when the constant steady state is unstable, and vice versa. In the case of instability, the periodic solution (48) thus furnishes the required estimate of the influence domain of the asymptotically stable constant steady state. In fact, if a differentiable $\theta(t)$ in (11), (30) is such that the curve segment defined parametrically by

$$
\phi(t)=\theta(t) \quad, \psi(t)=\dot{\theta}(t),-c \leqslant t \leqslant 0
$$

intersects on the phase cylinder the unstable limit cycle defined by (48), then the solution of (11), (30) will not approach the asymptotically stable state $\phi(t)=\phi_{1}$.

Now consider the equation

$$
\dot{x}(t)=-\left(\frac{\pi}{2}+a\right)\left[1+b x(t)+c x^{2}(t)\right] x(t-1)
$$

containing (35) and (36) as particular cases. The assumptions (39) are still appropriate, but instead of (48a) one finds

$$
A_{0}=\left(\frac{40 \alpha}{(3 \pi-2) b^{2}-5 \pi c}\right)^{\frac{1}{2}}, \quad h_{2}=\frac{b^{2} A_{0}^{2}}{10 \pi} .
$$

The periodic solution is asymptotically stable when $a>0$ and unstable when $a<0$.

For (37) the proper dependance on $\varepsilon$ is

$$
\begin{aligned}
& \tau=\tau_{0} t, \quad r_{0}=\sum_{i=0}^{\infty} h_{i} r^{i} \\
& g=\frac{\pi}{2}+\varepsilon\left(c_{0}+\bar{r}_{2} x^{2}(t)\right), x(t)=\sum_{i=0}^{\infty} \varepsilon^{i} x_{i}(\tau) .
\end{aligned}
$$

After inserting into (37) and proceeding as before, the absence of secular terms in $x_{1}(\tau)$ and $x_{2}(\tau)$ yields

$$
\begin{array}{ll}
A_{0}=\sqrt{\frac{-4 x_{0}}{3 \bar{x}_{2}}} \quad, \quad A_{1}=-\frac{\pi}{16} c_{0} A_{0} . \\
h_{1}=0 \quad, \quad h_{2}=\frac{1}{4} \kappa_{0}^{2} .
\end{array}
$$

In the original variables the periodic solution of (37) is

$$
\begin{aligned}
& x(t)=\left(\frac{4 c-2 \pi}{3 c_{2}}\right)^{\frac{1}{2}}\left[\left(1-\pi \frac{2 c-\pi}{32}\right) \cos \tau+\frac{c_{2}}{4}\left(\sin \tau-\frac{4}{3} \sin 3 \tau\right)+\cdots\right] \\
& \tau=\left(1+\frac{1}{16}(2 c-\pi)^{2}+\cdots\right)^{-1} \cdot t
\end{aligned}
$$

The solution (54) is found to be asymptotically stable when $c>\pi / 2$ and unstable when $c<\pi / 2$. If the delay $g$ in (52) is replaced by

$$
\begin{aligned}
g=\frac{\pi}{2}+\varepsilon\left[c_{0}\right. & +c_{1} x(t)+c_{2} x^{2}(t)+ \\
& \left.+c_{3} x^{3}(t)+c_{4} x^{4}(t)\right],
\end{aligned}
$$

then the generating amplitude is found to be

$$
A_{0}=\left[\frac{3 c_{2}}{5 c_{4}}\left(1 \pm \sqrt{1-\frac{40}{9} \frac{A_{0} c_{4}}{c_{2}^{2}}}\right)\right]^{\frac{1}{2}}
$$

Thus there exist two distinct periodic solutions when $0<40 \mathrm{c}_{0} \mathrm{c}_{4}<9 \mathrm{c}_{2}^{2}$ and a single one when $\mathrm{c}_{0} \mathrm{c}_{4}<0$. 
Asymptotically stable periodic solutions are possible even if $c_{0}<0$. When two distinct solutions exist, the one with the larger $A_{0}$ is asymptotically stable, while the other is unstable. The properties of (37) are similar to those of the Van der Pol equation.

The similarity between (37) and the Van der Pol equation suggest that one should look for a similarity between

$$
\begin{aligned}
& \dot{x}(t)=-x(t-g)-\varepsilon\left(b_{2} x^{2}(t)+b_{3} x^{3}(t)\right), \\
& g=\frac{\pi}{2}+\varepsilon \kappa_{0}
\end{aligned}
$$

and the Duffing equation. The magnitude assignments (52) are found to hold for (57), and one finds

$$
\begin{aligned}
& A_{0}=\sqrt{\frac{4 c_{0}}{3 b_{3}}}, h_{4}=0, h_{2}=\frac{a_{0}^{2}}{6}-\frac{b_{3}^{2}}{5} A_{0}^{2}, \\
& A_{1}=\left(\frac{\pi-2}{15} \frac{b_{7}^{2}}{b_{3}}-\frac{\pi+6}{24} c_{0}\right) A_{0},
\end{aligned}
$$

The first known examples of differential equations of second order, where the existence of periodic solutions has been ascribed to the presence of a small delay, are of the Van der Pol and Duffing type 22,23 )

$$
\begin{aligned}
& \ddot{x}(t)+a_{0} \dot{x}(t)+a_{1} x(t)+a_{2} \dot{x}(t-c)-\varepsilon \dot{x}^{3}(t-c)=0, \\
& \ddot{x}(t)+a \dot{x}(t)+x(t-c)+\varepsilon x^{3}(t-c)=0,
\end{aligned}
$$

respectively. The coefficients in (59), (60) are constants. The periodic solutions can be found in a straightforward manner if $|E| \ll 1$. As an illustration, consider $(60)$. The characteristic equation of its linear part is

$$
\gamma^{2}+a \gamma+e^{-c \gamma}=0
$$

If the value of $a$ is such that $c$ is close to its second bifurcation value $c_{1}$, i.e.

$$
a=\alpha+\varepsilon \beta, \quad \lim _{\varepsilon \rightarrow 0} \gamma= \pm i \omega, \omega>0,
$$

then the additional magnitude assignments are

$$
\omega t=\left(1+\sum_{i=1}^{\infty} k_{i} \varepsilon^{i}\right) \tau, x(t)=\sum_{i=0}^{\infty} \varepsilon^{i} x_{i}(\tau)
$$

Using (62), (63) the periodic solution becomes

$$
\begin{aligned}
x(t) & =A_{0} \cos \tau+\cdots, \tau=\left(1+h_{1} \varepsilon+\cdots\right)^{-1}, t \\
A_{0}= & {\left[\frac{4 \beta \omega(\alpha \sin \omega c+2 \cos \omega c)}{3 b \cos \omega c}\right]^{\frac{1}{2}}, h_{1}=\frac{\beta \omega}{b}, } \\
b= & (\alpha \sin \omega c+2 \cos \omega c) \operatorname{tg} \omega c-\alpha \omega-c \cos \omega c+ \\
& +2 \sin \omega c .
\end{aligned}
$$

The solution (64) is asymptotically stable when $b>0$ and $a-\alpha<0$, i.e. when the steady state $x(t) \equiv 0$ is unstable.

When $n=1, F(\phi)=$ sin $\phi$ the second-order phase lock equations (9), (10) become

$$
\begin{aligned}
\ddot{\phi}(t) & +\frac{1}{T_{1}}(1+k T \cos \phi(t)) \dot{\phi}(t)+\frac{k}{T_{1}} \sin \phi(t)= \\
& =\frac{1}{T_{1}} \omega(t)+\dot{\omega}(t)
\end{aligned}
$$

and

$$
\begin{gathered}
\ddot{\phi}(t)+\frac{1}{T_{1}} \dot{\phi}(t)+\frac{k T}{T_{4}} \dot{\phi}(t-c) \cdot \cos \phi(t-c)+\frac{k}{T_{4}} \sin \phi(t-\alpha)= \\
=\frac{1}{T_{1}} \omega(t)+\dot{\omega}(t),
\end{gathered}
$$

respectively, where $\mathrm{T}, \mathrm{T}_{1}$ are also positive constants.

If $\omega(t)=\omega_{0}$, the variational equation of $\phi(t)=\operatorname{arc}$ $\sin \omega_{0} / k$ is

$$
\ddot{\phi}(t)+\frac{1}{T_{1}} \dot{\phi}(t)+\frac{T}{T_{1}} \sqrt{k^{2}-\omega_{0}^{2}} \dot{\phi}(t-c)+\frac{1}{T_{1}} \sqrt{k^{2}-\omega^{2}} \phi(t-\alpha)=0 .
$$

Since the characteristic equation admits a bifurcation $c=c_{0}+E c_{1}$ producing purely imaginary roots $\gamma= \pm i \beta$, periodic solutions ascribable to a small delay are possible. The constant $\beta$ is a real root of

$$
T T_{1} \beta^{2}-\left(T+T_{1}\right) \beta \operatorname{tg} \kappa_{0} \beta-1=0 .
$$

The reduction of the influence domain of the steady state $\phi(t)=\phi_{1}$ due to the presence of an unstable periodic solution of (66) has already been observed, but detailed properties of the solutions of (67) are still unknown.

\section{NON-AUTONOMOUS PERIODIC STEADY STATES}

The study of periodic solutions of non-autonomous differential equations with delay is much more complex than that of autonomous ones, the main reason being the increase in the number of controlling parameters. In addition to those describing the nature of the nonlinearity, there appear at least three new ones: the strength (amplitude) of the "forcing signal", the order of the resonance (approximate ratio of the forcing and free frequencies), and the detuning (frequency deviation from the exact resonance). In the absence of a general existence theorem, it is indispensable to carry out many numerical computations before a class of solutions is found which falls into the scope of known analytical methods. Because of the numerous parameters, these computations are quite lengthy and their evaluation quite laborious. Without a preliminary numerical exploration, the analytical approximations are of doubtful value, and more often than not they turn out to be erroneous.

Non-autonomous differential equations with delay are not too well conditioned numerically, and the properties of their solutions appear to be more diverse than those of the corresponding generating differential equations (without delay). Because of these reasons, only fragmentary results on three particular equations of first order will be presented in this paper. These equations are (37), (38), and (57), modified by a purely sinusoïdal forcing term. The basic form corresponding to $(37),(38)$ is 


$$
\begin{aligned}
\dot{x}(t)= & -P\left(x_{t}\right) \cdot x(t-g)+\varepsilon^{i_{1}} \cdot \lambda \cos \left[\left(1+\varepsilon^{i_{2}} \delta\right) n t\right] \\
P\left(x_{t}\right)= & 1+b_{2} x(t-g)+b_{3} x^{2}(t-g) \\
g=\frac{\pi}{2}+ & \varepsilon^{i_{3}}\left[c_{0}+c_{1} x(t)+c_{2} x^{2}(t)+\right. \\
& \left.+c_{3} x^{3}(t)+c_{4} x^{4}(t)\right],
\end{aligned}
$$

where the constants $\lambda, n, \delta$ define the three controlling parameters of the forcing signal and $i_{j}$ are suitab1y chosen integers.

By analogy with what happens in the theory of ordinary differential equations, one would expect for (69) a certain continuity with respect to the parameter $\lambda$. In particular, since the small parameter method was found to apply under certain conditions when $\lambda=0$, under similar conditions it ought to apply when $0 \leq|\lambda| \ll 1$. Unfortunately, this argument turns out to be only partially successful. The obstacle encountered is of a computational nature. In fact, while it is relatively straightforward to compute asymptotically stable periodic solutions of $(69)$, it is much more difficult to compute unstable ones. In the absence of an (analytical) existence theorem it is therefore very hard to decide whether a lack of agreement between an analytical approximation and a corresponding numerical result is due to the fact that the solution sought for does not exist, or that it is merely unstable.

In principle it is possible to determine the stability of a particular periodic solution of (69) by means of its variational equation, because one does not deal with a Liapunov critical case when $\lambda \neq 0$, and it is known that the theory of Iinear differential equations with periodic coefficients and delay is similar to the theory of Floquet (cf. for example, Refs. $24-27$ and p. 51-78, Vol. 4 of Ref. 7), in the sense that there exist characteristic numbers from which stability or instability can be deduced. At present there are still numerous unsolved details in the determination of these characteristic numbers, which render the variational equation approach unwieldy, and the numerical exploration practically unavoidable.

\section{MAIN RESONANCE}

Let $\mathrm{n}=1$ and consider first the case $b_{2}=b_{3}=c_{3}=$ $\mathrm{c}_{4}=0$. The appropriate choice of the order of magnitudes is $i_{1}=i_{2}=i_{3}=1$ in $(69)$ and $i_{4}=0$ in

$$
x(t)=\sum_{j=0}^{\infty} \varepsilon^{j+i_{4}} \cdot x_{j}(t)
$$

Substituting (70) into (69) yields

$$
\begin{aligned}
& \dot{x}_{0}(t)+\varepsilon \dot{x}_{1}(t)+\cdots=\varepsilon \lambda \cos t-x_{0}\left(t-\frac{\pi}{2}\right)+ \\
& +\varepsilon \delta x_{0}\left(t-\frac{\pi}{2}\right)-\varepsilon\left[x_{1}\left(t-\frac{\pi}{2}\right)-\left(c_{0}+c_{1} x_{0}(t)+(71)\right.\right. \\
& \left.\left.+c_{2} x_{0}^{2}(t)\right) \dot{x}_{0}\left(t-\frac{\pi}{2}\right)\right] .
\end{aligned}
$$

Contrary to the autonomous case the equation

$$
\dot{x}_{0}(t)+x_{0}\left(t-\frac{\pi}{2}\right)=0
$$

now defines a generating solution with two undetermined constants

$$
x_{0}(t)=A \cos t+B \sin t .
$$

From (73) and the coefficient of $\varepsilon$ in (71) one finds

$$
\begin{aligned}
& \dot{x}_{1}(t)+x_{1}\left(t-\frac{\pi}{2}\right)=\frac{c_{1}}{2}\left(A^{2}+B^{2}\right)+ \\
& +\left[c_{0}+\frac{\pi \delta}{2}+\frac{3 c_{1}}{4}\left(A^{2}+B^{2}\right)+\lambda A^{-1}\right] A \cos t+ \\
& +\left[A_{0}+\frac{\pi \delta}{2}+\frac{3 c_{2}}{4}\left(A^{2}+B^{2}\right)+\delta A B^{-1}\right] B \sin t+ \\
& +\frac{c_{1}}{2}\left(A^{2}-B^{2}\right) \cos 2 t+c_{1} A B \sin 2 t+\cdots,
\end{aligned}
$$

The absence of secular terms in $\mathrm{x}_{1}(t)$ implies

$$
\begin{aligned}
& \frac{3}{4} c_{2} \delta^{2} \lambda^{-2} A^{5}+\frac{3}{4} \alpha_{2} A^{3}+\left(c_{0}+\frac{\pi \delta}{2}\right) A+\lambda=0 \\
& B=\delta \lambda^{-1} A .
\end{aligned}
$$

The algebraic equations (75) fix the real constants $A$ and $B$ uniquely for a wide range of $c_{0}, c_{2}, \delta$, and $\lambda$. For the exact resonance $(\delta=0)$ there results the explicitly solvable cubic equation

$$
\lambda^{3}+\frac{4 c_{0}}{3 c_{2}} A+\frac{4 \lambda}{3 c_{2}}=0, \quad B=0 .
$$

By comparing the "synchronized" generating amplitude $C=\sqrt{A^{2}+B^{2}}$ of (73) to the autonomous generating amplitude $A_{0}$ of (54) it is easily seen that $C$ increases with $\lambda$, i.e. the main resonance $\mathrm{n}=1$ of (37) is quite strong. The numerical computations confirm the existence of a synchronized periodic solution and show that for $c_{0}>0, c_{2}<0$ it is asymptotically stable in a wide range of $\delta$ and $\lambda$. This range is, however, smaller than the range of existence of real roots A, B of (75). For example; if $c_{0}=0.03$, $c_{2}=-0.1, \lambda=0.1$, then asymptotic stability exists in the interval $-0.07 \leq \delta \leq+0.07$, whereas real $\mathrm{A}, \mathrm{B}$ exist also for $|\delta|>0.07$ (sample comparison values are: $\mathrm{A}_{0}=0.6287, \mathrm{C}=\mathrm{A}=1.222$ if $\delta=0 ; \mathrm{C}=1.707$, $A=1.274, B=1.136$ if $\delta=0.07 ; \quad C=1.897$, $\mathrm{A}=1.209, \mathrm{~B}=1.461$ if $\delta=0.1$; the corresponding numerically determined amplitudes

$$
\begin{gathered}
C_{n}=\frac{1}{2}[\max x(t)-\min x(t)] \\
t_{0} \leqslant t \leqslant t_{0}+2 \pi(1+a)^{-1}
\end{gathered}
$$

are: $C_{n}=1.220$ if $\delta=0 ; C_{n}=1.332$ if $\delta=0.07$; none if $\delta=0.1$ ). For $\lambda=0.01$, the synchronization interval diminishes to $-0.01<\delta<+0.01$.

The variational equation of (69) corresponding to (73) is

$$
\begin{aligned}
& \dot{y}(t)+2 \frac{\varepsilon c_{2}}{1+2 \delta}\left[x_{0}\left(t-g_{0}\right)\right] \cdot y(t)+\frac{1}{1+\varepsilon \delta} y\left(t-g_{0}\right)=0 \\
& g_{0}=\frac{\pi}{2}+\varepsilon\left[c_{0}+t_{1} x_{0}(t)+c_{2} x_{0}^{2}(t)\right] .
\end{aligned}
$$

The solutions of (76) are unfortunately still unknown. Synchronized periodic solutions of (69) exist also when $c_{4} \neq 0$, but their properties are too varied to be described briefly. 
(21) N. Krylov and N. Bogoliubov, Introduction to non-linear mechanics (ONTI, Kiev, 1937), p. 106. This transformation is also described in most books on quasi-linear oscillations. An example is: I.G. Malkin, Some problems of the theory of non-linear oscillations (GOSISTEKHTEOLIT, Moscow, 1956).

(22) N. Minorsky, CR Acad. Sci. 226, 122 (1948).

(23) E. Leimanis and N. Minorsky, Dynamics of nonlinear mechanics (John Wiley and Sons, Inc., New York, 1958), p. 176.

(24) A.M. Zverkin, Dokl. Akad. Nauk SSSR, 128, 882 (1959).

(25) A. Halanay, Revue de Math, pures et appl. $\underline{6}, 141$ (1961).
(26) A.A. Stokes, Proc. Nat. Acad, Sci. (USA) 48, 1330 (1962).

(27) S.N. Shimanov, Prik1. Mat. Mekh. 27, 450 (1963).

(28) I.G. Malkin, Some problems of the theory of nonlinear oscillations (GOSISTEKHTEOLIT, Moscow, 1956).

(29) V.I. Zubov, Oscillations in non-linear and regulated systems (SUDPROMGIZ, Leningrad, 1962).

(30) J.K. Hale, Oscillations in non-linear systems (McGraw-Hill, Inc., New York, 1963) and J. Differential Equat. 2, 57 (1966).

(31) M.A. Krasnoselskii, V.Sh. Burd and In.S. Kolesov, Non-linear almost-periodic solutions, Nauka (Moscow), 1970.

Table 1

\begin{tabular}{|l|l|l||c|c|c|c|c|c|}
\hline$\delta$ & $-\alpha$ & $\beta$ & $\delta$ & $-\alpha$ & $\beta$ & $\delta$ & $-\alpha$ & $\beta$ \\
\hline 0 & 1 & 0 & 0.5 & 1.58805 & 1.54022 & 1.5 & 0.02186 & 1.03310 \\
0.1 & 1.11833 & 0 & 0.6 & 1.11715 & 1.60413 & 1.52 & 0.01538 & 1.02354 \\
0.2 & 1.29586 & 0 & 0.7 & 0.80696 & 1.56323 & 1.54 & 0.00914 & 1.01414 \\
0.3 & 1.63134 & 0 & 0.8 & 0.59120 & 1.49187 & 1.56 & 0.00315 & 1.00491 \\
0.36 & 2.23912 & 0 & 0.9 & 0.43492 & 1.41371 & 1.568 & 0.00081 & 1.00127 \\
0.366 & 2.46495 & 0 & 1.0 & 0.31813 & 1.33724 & 1.57 & 0.00023 & 1.00036 \\
$1 / \mathrm{e}$ & $\mathrm{e} \approx 2.71828$ & 0 & 1.1 & 0.22866 & 1.26549 & $\pi / 2$ & 0 & 1 \\
0.368 & 2.71680 & 0.06956 & 1.2 & 0.15872 & 1.19935 & 1.572 & -0.000347 & 0.99946 \\
0.374 & 2.64437 & 0.48528 & 1.3 & 0.10314 & 1.13883 & 1.574 & -0.000921 & 0.99855 \\
0.4 & 2.36022 & 1.01817 & 1.4 & 0.05836 & 1.08356 & 1.576 & -0.00149 & 0.99765 \\
\hline
\end{tabular}

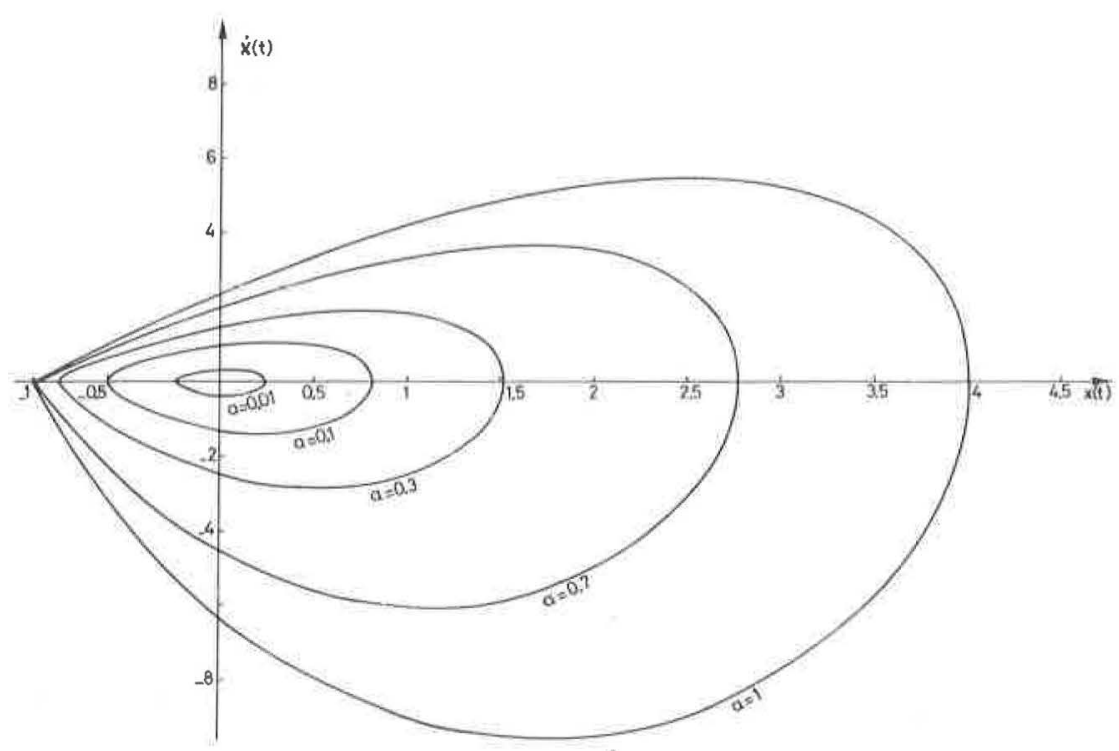

Fig. $1 \quad \dot{x}(t)=-\left(\frac{\pi}{2}+a\right)[1+x(t)] x(t-1)$

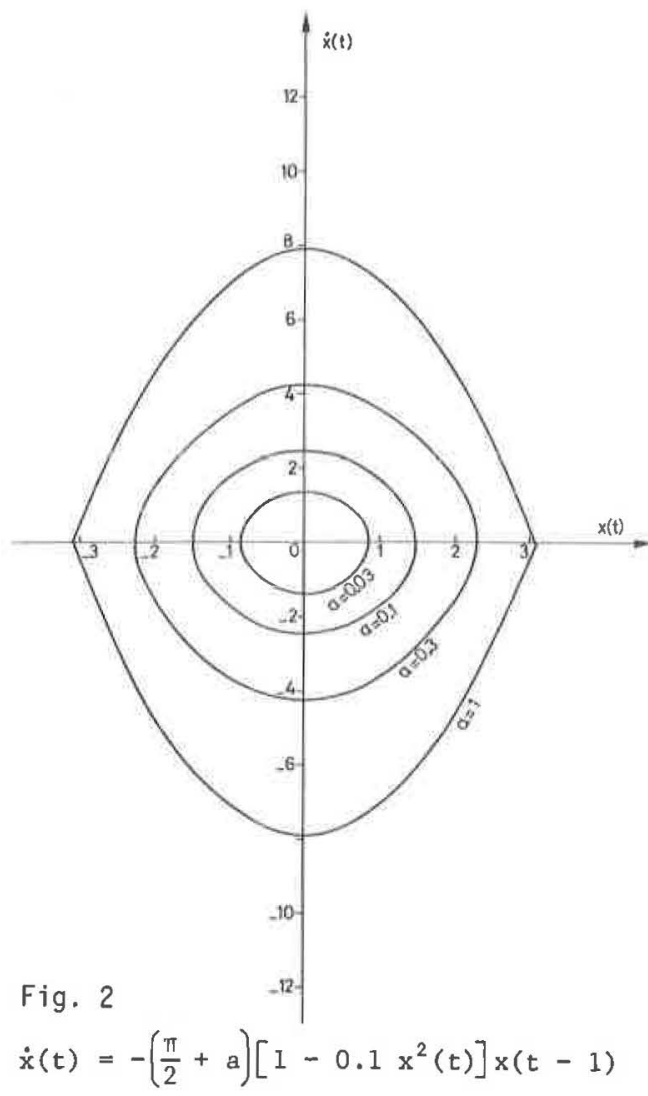




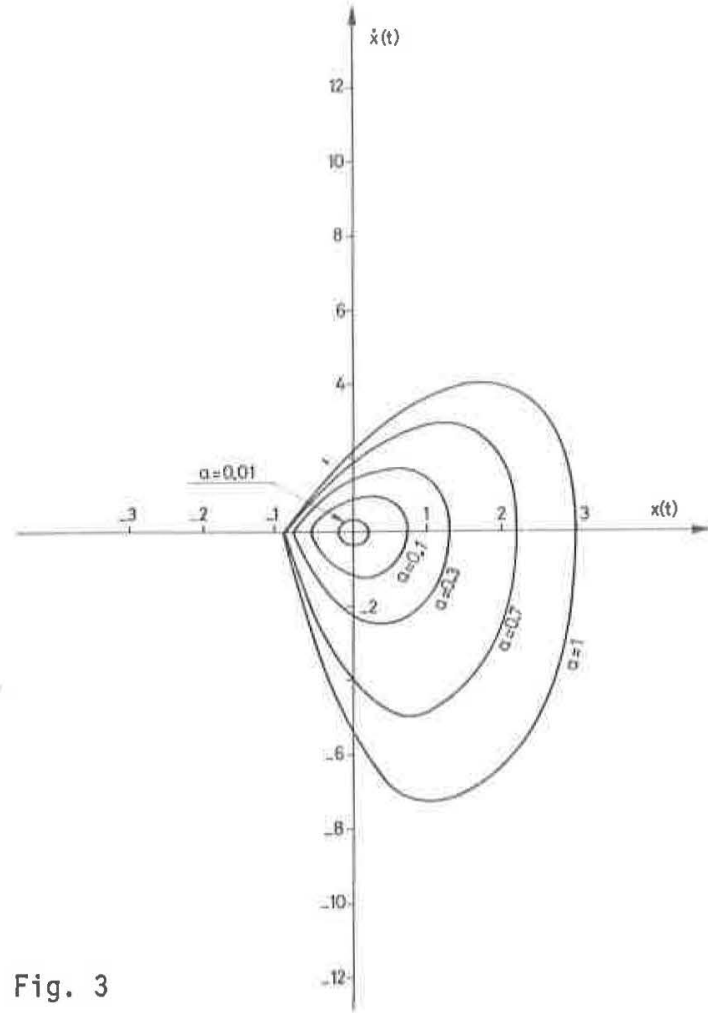

$\dot{x}(t)=-\left(\frac{\pi}{2}+a\right)\left[1+x(t)-0.1 x^{2}(t)\right] x(t-1)$
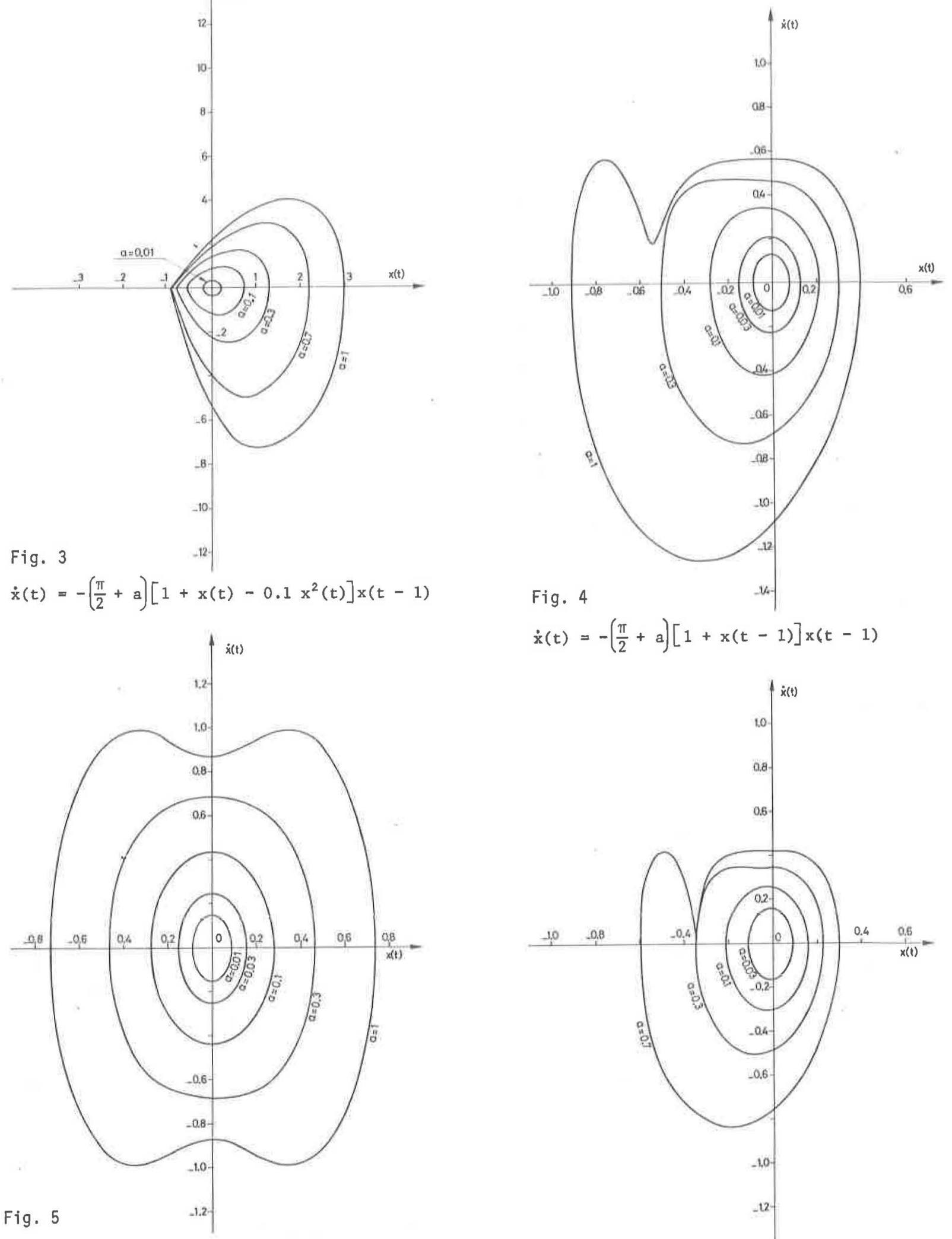

$\dot{x}(t)=-\left(\frac{\pi}{2}+a\right)\left[1-x^{2}(t-1)\right] x(t-1)$

$\dot{x}(t)=-\left(\frac{\pi}{2}+a\right)[1+x(t-1)] x(t-1)$

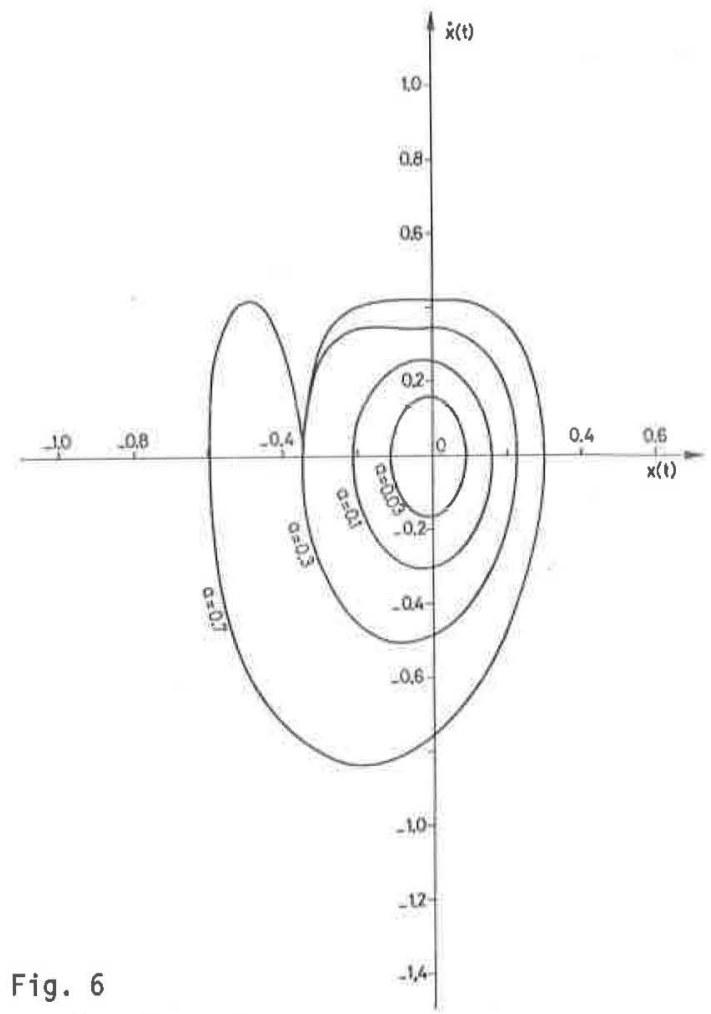

$\dot{x}(t)=-\left(\frac{\pi}{2}+a\right)\left[1+x(t-1)-x^{2}(t-1)\right] x(t-1)$ 
Fig. 7

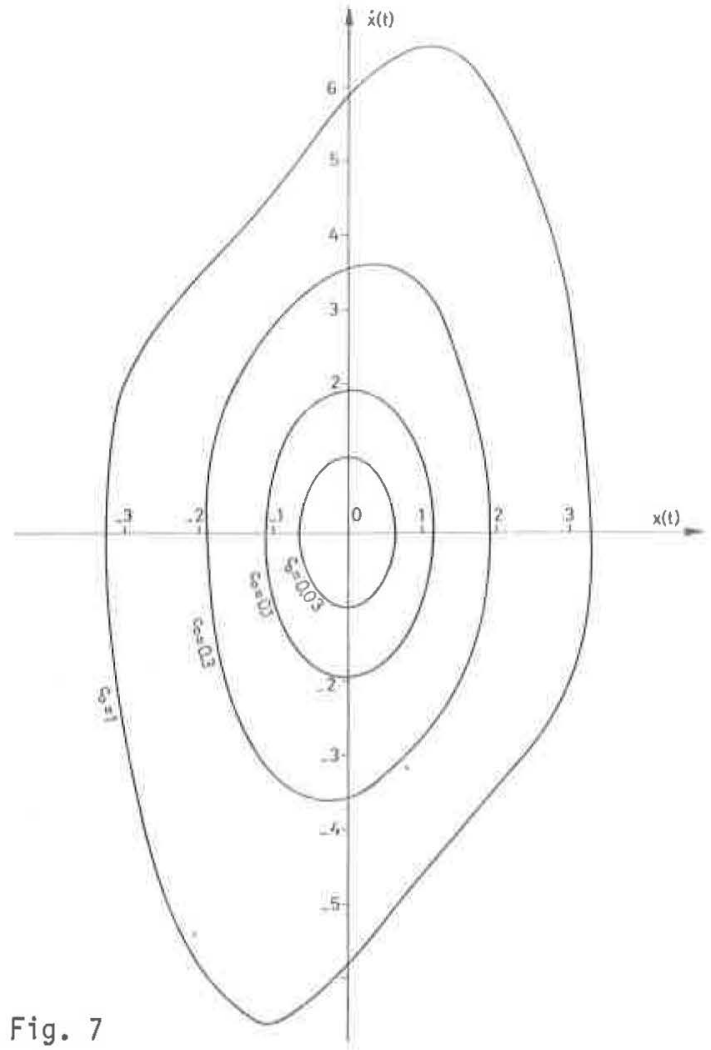

$\dot{x}(t)=-x(t-g), g=\frac{\pi}{2}+c_{0}-0.1 x^{2}(t)$

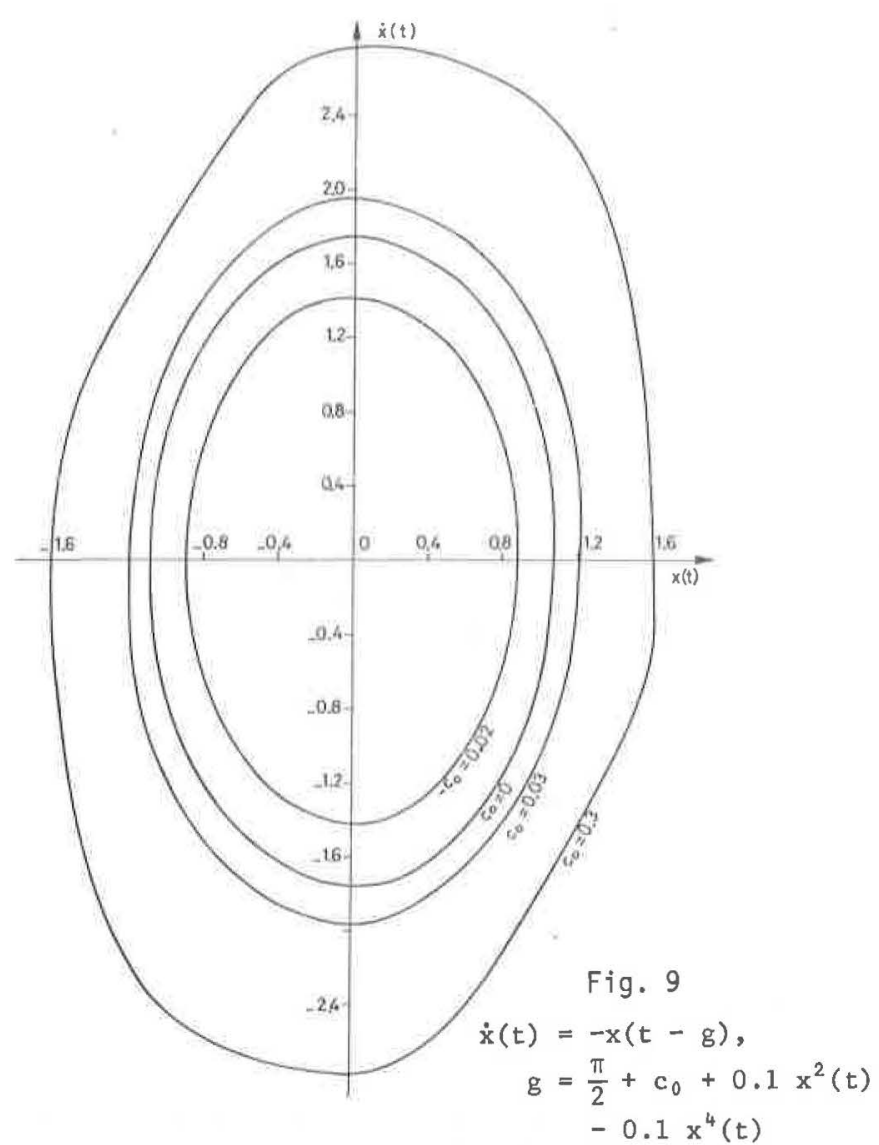

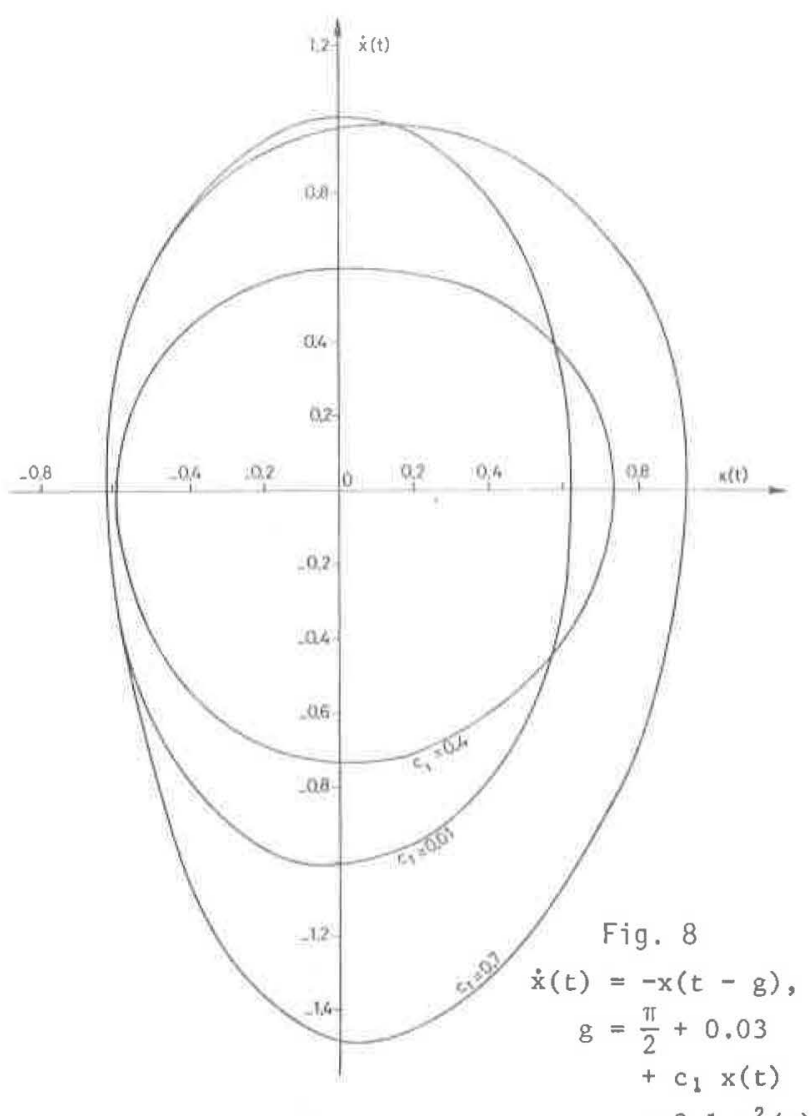

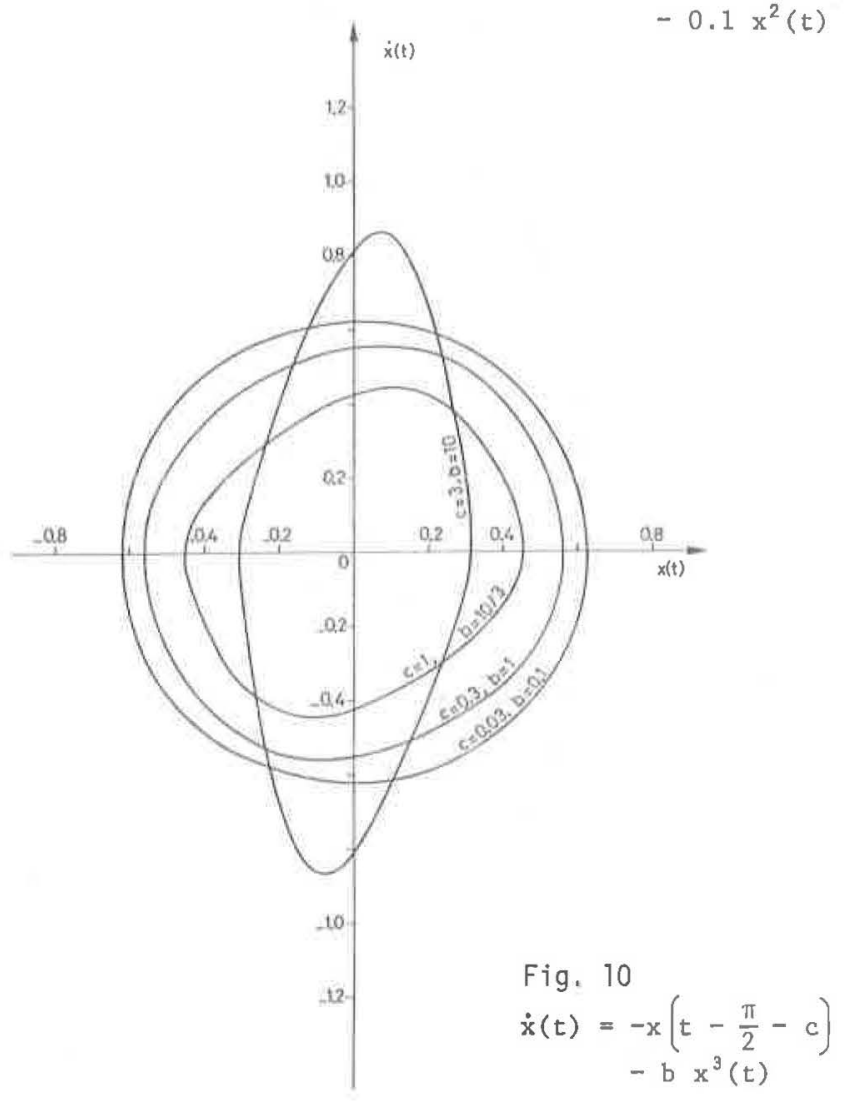

